\title{
Exploration evoked by electrical stimulation of the amygdala of rats
}

\author{
HARVEY WEINGARTEN and NORMAN WHITE \\ McGill University, Montreal, Quebec H3A IB1, Canada
}

\begin{abstract}
Food-deprived rats were placed individually into a novel test cage containing food pellets for $5 \mathrm{~min}$ per day for 4 days. Detailed observation of their behavior showed that the rats ate more and explored less each day, and that there was a consistent pattern of the component behaviors of exploration in all rats. Over the next 5 days, the same experimental procedure was followed, except that the rats were given low-level stimulation of the basolateral area of the amygdala. During the stimulation phase, the rats exhibited the same patterns of behavior as they did during the nonstimulation phase, and the components of evoked exploration were highly similar to those observed during normal exploration. Additional groups of rats stimulated in the lateral or ventromedial areas of the hypothalamus exhibited behavior patterns which did not resemble the patterns of normal or amygdaloid-stimulation-evoked exploration. The data suggest that the amygdala may function as a part of a neural circuit which mediates the exploration that occurs in response to novelty. This would account for the well-known finding that amygdaloid stimulation interrupts various appetitive behaviors.
\end{abstract}

Although it is well known that stimulation of the amygdala can suppress feeding (Grossman \& Grossman, 1963; White, 1973; White \& Fisher, 1969) and other motivationally relevant behaviors (Goddard, 1964; Kaada, 1972), the implications of this finding for an understanding of the role played by the amygdala in the mediation of normal behavior remain obscure. Kaada (1972) interprets these data as showing that the amygdala can exert "a tonic, inhibitory influence on a number of activities"; and Goddard (1964) concluded, more specifically, that the amygdala "suppresses motivated approach behavior."

These interpretations of the effects of amygdaloid stimulation focus upon the fact that feeding (or some other activity) is interrupted, but give no attention to what the animals do instead of eating while they are stimulated. An analysis based on the behavior that occurs during stimulation (instead of on the behavior that is interrupted) would provide a different focus for understanding these aspects of amygdaloid function, provided that the behavior evoked by the stimulation is itself interpretable as some form of organized behavior. In a previous paper, White (1973)

The research reported here was supported by grants from the National Research Council of Canada and from FCAC, Department of Education, Province of Quebec. We thank Garry Bernstein and the computer laboratory of the McGill Psychology Department for developing the program used for the behavior observations. We thank Dr. Richard Davies and Daniel Coulombe for critical comments on an earlier version of the manuscript. Harvey Weingarten is presently at the Department of Psychology, Yale University. Requests for reprints should be sent to Norman White, Department of Psychology, McGill University, 1205 McGregor Avenue, Montreal, Quebec H3A 1B1, Canada. made the casual observation that, during stimulation of the amygdala, rats which had interrupted their feeding appeared to explore the test cage. In the experiment reported here, we examine the possibility that some form of normal exploration actually does occur during amygdaloid stimulation. To do this, we made formal observations of normal and stimulation-evoked behavior and compared the resulting patterns statistically.

Interruption of feeding has also been reported during stimulation of the lateral hypothalamus ( $\mathrm{LH}$ : Christopher \& Butter, 1968) and the ventromedial hypothalamus (VMH: Anand \& Brobeck, 1951; Krasne, 1962; Lewinska \& Romaniuk, 1966; Smith, 1956). In the case of the LH, Christopher and Butter (1968) and Valenstein, Cox, and Kakolewski (1968) observed a behavior they described as "exploratory forward locomotion"'; and in the case of the VMH, Krasne (1962) and Lewinska and Romaniuk (1966) described "disruption," which they attributed to stimulation-produced aversion. Because of these reports, it seemed useful to include an analysis of the behavior that occurs during interruptions of feeding produced by stimulation of these two brain sites for comparison with the amygdaloid data.

According to the theoretical view of the neural organization of motivated behavior proposed by Glickman and Schiff (1967), if electrical stimulation of a particular brain site evokes normal, speciesspecific behavior, electrical self-stimulation should also be observable at that site. Self-stimulation has, in fact, been reported with electrode placements in the amygdala (White, 1973; Wurtz \& Olds, 1963), the LH (Hoebel \& Teitelbaum, 1962; Margules \& 
Olds, 1962), and the VMH (Ball, 1972; Reynolds, 1958). To gain additional information about the nature of the behavior evoked by stimulation of these three brain areas, all subjects were also tested for self-stimulation at the same stimulation parameters that were used to study evoked behaviors.

\section{METHOD}

\section{Subjects}

The subjects were 16 male hooded rats weighing $250-300 \mathrm{~g}$ at the time of surgery. They were housed two to a cage with water ad lib, and food (Purina Lab Chow pellets) available according to the experimental protocol.

\section{Apparatus}

The experimental rooms were maintained on a $12 \mathrm{~h}: 12 \mathrm{~h}$ reverse day-night cycle, and all experimental manipulations began $2 \mathrm{~h}$ after the start of the dark period. The observation cage consisted of a rectangular $(54 \times 54 \times 40.5 \mathrm{~cm})$ cage with a wood floor and Plexiglas walls. A commutator mounted over the center of the cage allowed an animal free access to all parts of the observation cage when its electrode was connected to the stimulator.

The stimulation electrodes were bipolar; each electrode was made from two lengths of insulated stainless steel wire twisted together. Sine-wave stimulation was obtained from a stimulator on which train duration and current intensity were independently adjusted.

Time sampling was carried out with the aid of a PDP-11 computer programmed to distinguish up to 10 different behaviors and to provide various analyses of the overall behavior patterns of the rats. There were seven mutually exclusive behavior categories which had been determined in preliminary studies to account for all of the behavior exhibited by rats in the test cage used in the experiment. These behaviors are listed in Table 1 along with a brief description of each.

The self-stimulation test took place in a wooden box $(20.5 \times$ $20.5 \times 40.0 \mathrm{~cm}$ ) with a Plexiglas front. A Plexiglas bar protruded into the cage through a side wall.

Table 1

Categories for Behavior Observations

Behavior Description

\section{Total Exploration}

Rearing

Motionless Sniffing Rat sits motionless but sniffs

Walking

Grooming

Feeding

Freezing

Escape
Rat rears on hind legs; usually accompanied by sniffing

Rat walks and changes position so as to involve all four limbs, usually accompanied by sniffing

Rat licks, scratches, or cleans any part of its body

Rat chews or nibbles food pellets

Rat sits or stands rigidly motionless in a hunched or prone position, tail elevated off floor, attentive and alert (seen only with stimulation of the amygdala)

Rat attempts to jump out of cage or darts from corner to corner and cowers there (seen only with stimulation of the VMH)

\section{Procedure}

All animals underwent surgery to implant a stimulating electrode using standard stereotaxic procedures and sodium pentobarbital $(50 \mathrm{mg} / \mathrm{kg})$ anesthesia. In six animals, implants were aimed at the basolateral amygdala (4.2, 5.2, -3.0; de Groot, 1959), in four animals they were aimed at the lateral hypothalamic nucleus $(6.0,2.0,-2.0)$, and in six animals they were aimed at the ventromedial nucleus of the hypothalamus $(6.0,0.6,-3.0)$. Following surgery, a $0.3-\mathrm{cc}$ prophylactic dose of procaine penicillin was injected intramuscularly and the animals were allowed a 5-day recovery period with food and water available ad lib.

After recovery, the rats' food was removed from their home cages, and, for 7 days, they were given access to food pellets in their home cages for $1 \mathrm{~h}$ per day, beginning $2 \mathrm{~h}$ after the start of the dark period. The behavioral observations began on the 8th day of food deprivation. On the behavior-observation days, the 1-h home cage feeding continued, following the observation period for each rat by 15 to $30 \mathrm{~min}$.

On each of the first 4 behavioral observation days, the animals were placed into the observation cage one at a time. The cage contained a petri dish filled with food pellets. The behavior of each rat was recorded for 5 min. During this period, each rat's electrode was connected to the commutator, but the stimulator was not turned on.

On the day following the 4 nonstimulation observation days, current thresholds were determined. Once a spontaneous bout of eating in the observation cage had begun, the stimulator was turned on at a subthreshold current level. The current level was then slowly increased until the threshold for the desired behavior change was reached. In the case of both the BLA and VMH animals, this consisted of the minimum current at which the animal stopped eating. For animals with electrodes in the $\mathrm{LH}$, the threshold was the minimum current which produced forward locomotion. This procedure was repeated until two successive determinations gave identical threshold current values. Once a current threshold had been determined for an animal, it received that stimulation current during all subsequent testing, both in the observation cage and in the self-stimulation tests.

For the next 5 days, Days 6-10 inclusive, the animals were tested in a manner identical to that of the first 4 nonstimulation days, except that each animal received continuous electrical stimulation of the brain during its 5-min abservation period.

Following the final observation day, the animals were returned to an ad-lib feeding schedule for 1 week and were then tested for electrical self-stimulation of the brain. The rats "selfadministered" trains of brain stimulation at their predetermined current levels. The durations of the trains were determined by the durations of the barpresses. Most rats required "shaping" to produce the required barpressing response. Presumably, this was because of the long trains of noncontingent stimulation which these rats had received during the observation tests. Self-stimulation testing continued until an animal's response rate was steady for $30 \mathrm{~min}$, at which time measures of barpressing frequency and duration were recorded.

Following the completion of all testing, each animal was given an overdose of chloral hydrate and was perfused intracardially with normal saline followed by $10 \%$ buffered Formalin. The brains were removed and sliced on a freeze microtome at $40 \mu$; relevant histological sections through the electrode tracts were mounted and stained with cresyl violet.

\section{RESULTS}

The position of the tip of each rat's electrode is shown in Figure 1. The median stimulation current levels used were $50 \mu \mathrm{A}$ (range $=25-55 \mu \mathrm{A}$ ) for the LH group; $20 \mu \mathrm{A}$ (range $=12-22 \mu \mathrm{A})$ for the $\mathrm{VMH}$ 
- Basolateral Amygdala

- Lateral Hypothalamus

- Ventromedial Hypothalamus

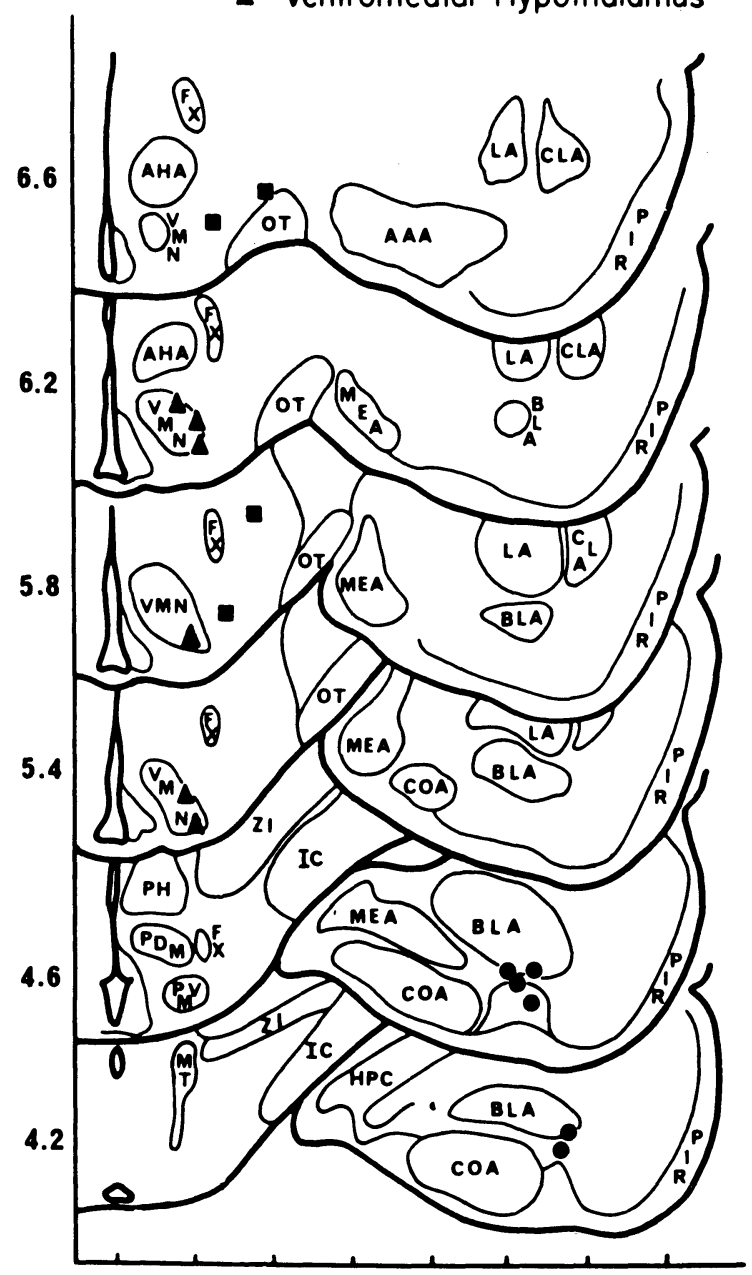

Figure 1. Locations of the tips of the stimulating electrodes. The sections are based on the atlas of de Groot (1959). The numbers on the left of the figure indicate the anterior-posterior plane of each section. Abbreviations: AAA, anterior amygdaloid area; AHA, anterior hypothalamic area; BLA, basolateral amygdala; CLA, claustrum; FX, fornix; HPC, hippocampus; IC, internal capsule; LA, lateral amygdaloid nucleus; MEA, medial amygdaloid nucleus; MT, mammillothalamic tract; OT, optic tract; PDM, posterior dorsal mammillary nucleus; $\mathbf{P H}$, posterial hypothalamus; PIR, pyriform cortex; PVM, posterior ventral mammillary nucleus; VMN, ventromedial nucleus; ZI, zona incerta.

group; and $19 \mu \mathrm{A}$ (range $=15-22 \mu \mathrm{A}$ ) for the BLA group.

An overall summary of the behavioral data is shown in Figure 2. A two-way analysis of variance was computed for feeding time and for exploration time in each of the two phases of the study (nonstimulation, Days 1-4; and stimulation, Days 6-10). The two factors in the analyses were groups and days (repeated measures), so that they assess any difference among the means for the three groups, any sustained day-to-day changes, and any difference among the groups in these day-to-day changes (interaction term).

During the first 4 nonstimulation days, there were no significant differences among the groups in feeding times $[\mathrm{F}(2,13)=2.28]$ or in total exploration times $[F(2,13)=1.46]$. However, there were significant day-to-day changes in both of these behaviors [feeding: $F(3,39)=49.04, p<.01$; exploration: $F(3,39)=28.29, p<.01]$. There were no significant interactions. Figure 2 shows that, as exploration decreased, feeding increased in all three groups.

During the 5 stimulation days, there was a significant difference among the groups in total exploration times $[\mathrm{F}(2,13)=4.56, \mathrm{p}<.05]$ and in feeding times $[F(2,13)=8.79, p<.01]$. There were no significant days effects in these analyses, but the interactions were significant [exploration: $F(8,52)=2.40$, $\mathrm{p}<.05$; feeding: $F(8,52)=8.00, p<.01]$. Figure 2 shows that the behavior of the BLA group accounts for these significant effects on both measures. The day-to-day release of feeding suppression in this group, which resembled the behavior of the unstimulated animals on Days 1-4, was in marked contrast to the sustained suppression in the VMH and LH groups. The same relationships can be seen in the patterns of exploration, although, as would be expected, they are in the opposite direction.

Stimulation of the amygdala produced an overall pattern of behavior which was similar to that observed during adaptation to a novel environment; stimulation of $\mathrm{VMH}$ and $\mathrm{LH}$ did not. A more detailed examination of these differences was made by considering the three component behaviors of the total exploration score and, in addition, two behaviors that were observed only during the stimulation phase of the study-escape for the VMH group and freezing for the BLA group. An examination of Figure 3 shows that stimulation of each brain area did, in fact, evoke a different pattern of these behaviors. These evoked behavior patterns were characterized statistically and descriptively by comparing each of them to the behavior patterns observed during the normal adaptation period in the nonstimulation part of the experiment using a set of two-way analyses of variance. Nonstimulation vs. stimulation was one factor in the analyses, and days was the other factor.

During stimulation of the BLA, the rats spent most of their time engaged in motionless sniffing with lesser amounts of walking, rearing, and freezing. In comparison with their behavior during the nonstimulation sessions, motionless sniffing $[F(1,5)=$ $62.29, \mathrm{p}<.01$ ] was significantly increased and freezing was introduced. The absence of any other significant group, day, or interaction effects between the nonstimulation and stimulation phases of the 


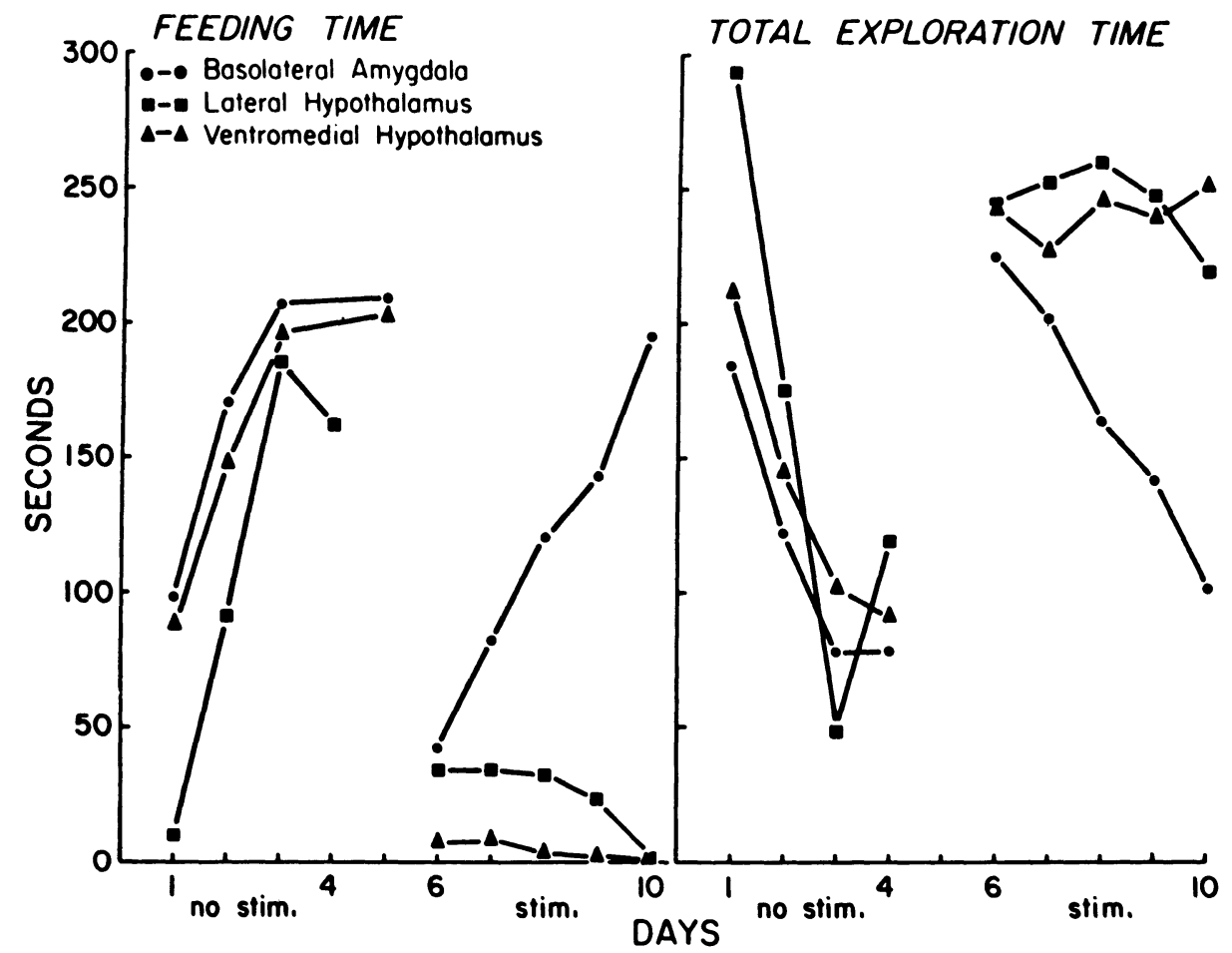

Figure 2. Mean daily values of time spent feeding and time spent exploring for rats in the three experimental groups.

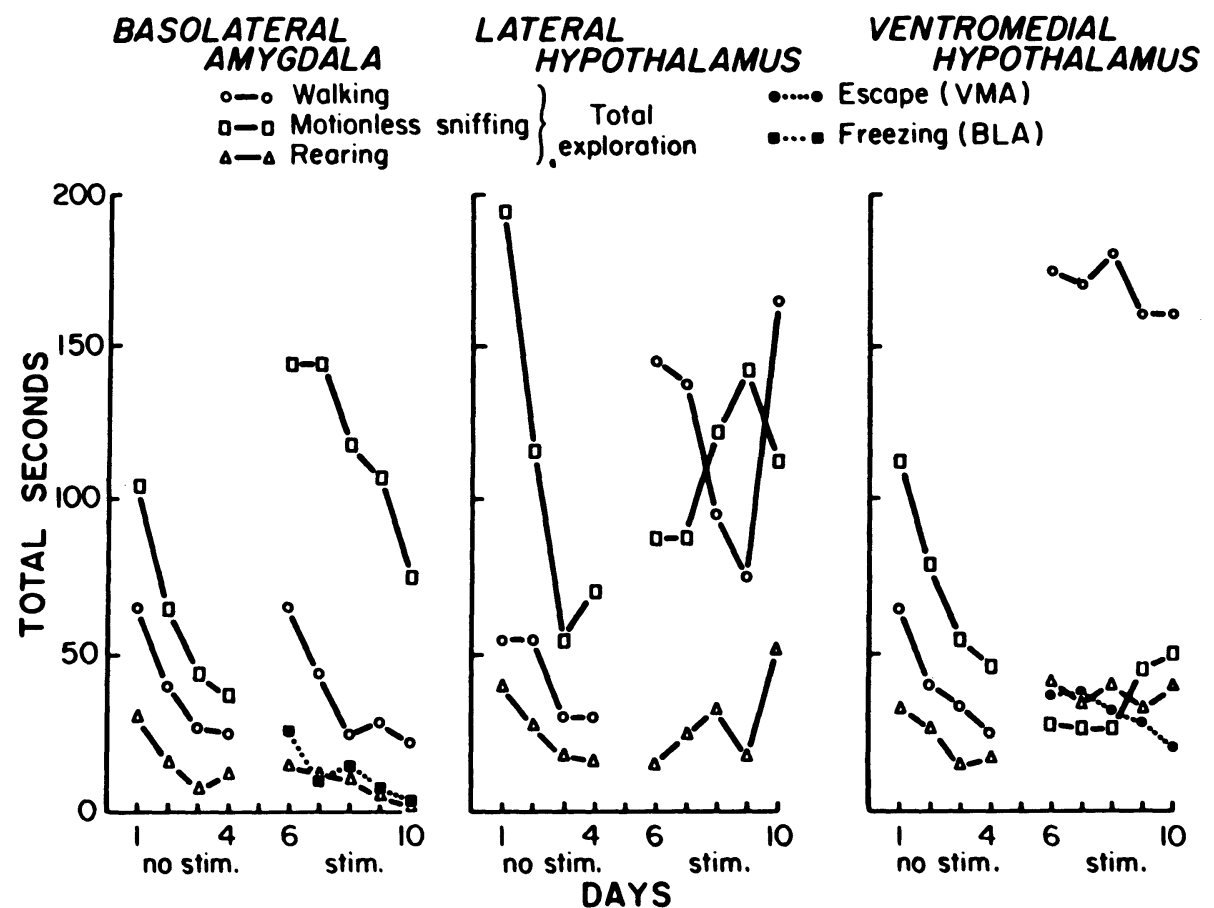

Figure 3. Mean daily time spent engaged in various behaviors for rats in the three experimental groups. 
experiment is suggestive of the high degree of similarity in the animals' behavior in both phases. Although there was some freezing and a relatively large amount of motionless sniffing during the stimulation phase (Figure 3), the three components of exploration continued to occur together so that the overall pattern of normal exploratory behavior was maintained.

For stimulation of the LH group, the apparently equal amounts of motionless sniffing and walking are misleading, as these means represent the fact that one of the four rats spent almost the entire observation time on all days engaged in motionless sniffing, while the other three rats exhibited a steretotyped pattern of behavior, which consisted of an orderly progression from one corner of the cage to another in a direction contralateral to the side of the brain being stimulated. At each corner, these rats reared, sniffed, and then proceeded to the next corner. For each rat, these stereotyped behavior patterns remained unchanged during the five stimulation sessions. In comparing the behavior of these rats during stimulation with their behavior during the nonstimulation sessions, the analyses showed that there were no significant differences in the overall levels of any of the behaviors during the two phases. However, a significant interaction was found for motionless sniffing $[F(3,9)=12.76, p<.01]$. Although the amount of this behavior decreased during the rats' first 4 days of exposure to the novel environment, the stereotyped nature of their behavior during the stimulation days resulted in a slightly increasing amount of motionless sniffing over the 5 days.

During VMH stimulation, the rats spent most of the time walking rapidly around the test cage, punctuating this behavior with very rapid movements and attempts to jump over the sides of the cage (escape). In comparing this behavior pattern to the behavior of the same rats during the nonstimulation phase, the analysis of variance showed that the amount of walking was significantly increased $[F(1,5)=37.78$, $\mathrm{p}<.01$ ], while the amount of motionless sniffing was significantly decreased $[F(1,5)=45.24, p<.01]$. There was also a significant interaction for motionless sniffing $[\mathrm{F}(3,15)=6.79, \mathrm{p}<.01]$. This occurred because the amount of this behavior decreased from day to day during the nonstimulation period, but increased over Days 6-10.

Data on the self-stimulation tests are given in Table 2. All subjects tested in the BLA and LH groups exhibited steady rates of responding, and the amount of stimulation they received was clearly well above operant rates determined by previous testing of a group of nonstimulated rats in the apparatus. The rats in the LH and BLA groups both exhibited stimulation-induced movement while the bar was depressed and the current was on. This movement took longer to develop in the BLA than in the $\mathrm{LH}$ rats
Table 2

Summary of Self-Stimulation Tests

\begin{tabular}{lccc}
\hline Group & $\begin{array}{c}\text { Mean } \\
\text { Bumber of } \\
\text { Barpresses/ } \\
\text { Min }\end{array}$ & $\begin{array}{c}\text { Mean Duration } \\
\text { of Stimula- } \\
\text { tion Trains } \\
\text { (Sec) }\end{array}$ & $\begin{array}{c}\text { Mean Amount } \\
\text { of Stimula- } \\
\text { tion/Min } \\
\text { (Sec) }\end{array}$ \\
\hline OP* & .2 & 1.7 & .7 \\
BLA & 7.7 & 3.5 & 26.7 \\
LH & 9.0 & 1.8 & 16.3 \\
VMH & .7 & .4 & .3 \\
\hline
\end{tabular}

*Operant rate $(N=4)$

but, in both cases, the movement caused the rats to leave the bar, turning off the stimulation. None of the VMH rats self-stimulated, and the total amount of stimulation received by these rats was actually somewhat lower than the operant level.

\section{DISCUSSION}

The behavior pattern exhibited by the rats in all three groups during the nonstimulation phase of the experiment was a form of exploration. In the present case, the pattern can be identified as "noveltyadjustive" (McReynolds, 1962), or "fear-related" (Halliday, 1966), since the rats were placed in a novel environment with no possibility of escape. Of the three stimulation groups, only the behavior of the BLA rats resembled this novelty/fear pattern. It is unlikely that the day-to-day decrease in the effectiveness of the BLA stimulation (Figure 2) was due to gross pathological changes in the stimulated tissue, because this was the only one of the three stimulated areas to exhibit this phenomenon, because the behavior pattern evoked was well integrated and organized even as it diminished in frequency over the stimulation sessions, and because the rats self-stimulated after the completion of the stimulation-observation sessions. It is also unlikely that the phenomenon of "kindling"' (Goddard, McIntyre, \& Leech, 1969) was involved in this change, because the current levels used were considerably lower than those required to produce this effect (Racine, 1972). However, the behavioral data do suggest that some progressive change in the neural substrate mediating the effects of the stimulation occurred over the five stimulation sessions.

The individual components of the behavior of the BLA rats (Figure 3) were, on the whole, similar in the two phases of the experiment. However, the stimulation phase differed from the nonstimulation phase in two ways: there was an elevated amount of motionless sniffing, and freezing was introduced. According to Barnett (1963), Berlyne (1966), Halliday (1966), McReynolds (1962), Montgomery (1955), and Welker (1959), these behaviors are characteristic of 
rats' responses to novelty/fear situations. Thus, during the nonstimulation phase, the rats' behavior met the operational criterion for novelty/fear exploration and, during the stimulation phase, their behavior met a descriptive criterion for the same type of behavior.

The fact that an apparently normal behavior pattern was observed during the wholly abnormal conditions of activation of the amygdala that were undoubtedly produced by the stimulation requires some comment. Even though it is unlikely that the amygdala itself was in a normal state during the stimulation, the exaggerated efferent activity produced by the activation of this structure apparently biased a neural system in such a way that the animals responded to their surroundings with a behavior pattern resembling that normally produced in novelty/ fear situations. The normally occurring day-to-day changes in this response pattern were also present during the stimulation phase, strengthening the interpretation that amygdaloid efferent activity is associated with a neural bias to respond in this manner.

The idea that activation of the amygdala may produce patterns of limbic neural activity leading to the characteristic response pattern in novelty/fear situations is consistent with studies showing that lesions of this structure reduce the behavioral signs of fear displayed by rats placed into fear-producing situations (Blanchard \& Blanchard, 1971, 1972; Kemble \& Tapp, 1968; Pellegrino, 1968). Amygdaloid lesions would, according to the present interpretation, eliminate or reduce the source of the neural bias that produced this pattern of responding. Moreover, the self-stimulation data of the present study suggest that the pattern evoked by the stimulation was, in some way, an adaptive one (Glickman \& Schiff, 1967). Therefore, we suggest that activation of the amygdala in the present study interrupted feeding because it increased the probability of occurrence of a precedent behavior pattern, that is, the one normally produced by novel, fear-producing situations.

Stimulation of the $\mathrm{LH}$ produced behavior patterns which were clearly different from those produced by stimulation of the amygdala, although the size of the sample makes conclusions difficult. Three of the four rats in this group exhibited mostly walking, a behavior that was apparently similar to what Christopher and Butter (1968) called "locomotor exploration," which they also observed during lateral hypothalamic stimulation. Christopher and Butter rejected the possibility that the stimulation was aversive, because the rats in their experiment selfstimulated at the same parameters that produced locomotion. The same argument applies to the present study. Moreover, a comparison of the behavior of the LH rats with that of the VMH rats, which included obvious attempts to escape from the cage, shows obvious differences which make the aversion hypotheses unlikely. The behavior produced by LH stimulation in Christopher and Butter's study and in the present study also clearly fails to resemble the novelty/fear exploration pattern of Phase 1 , and a comparison of these LH patterns with those observed in novelty-seeking situations (Montgomery, 1955; Welker, 1959) also reveals a complete lack of resemblance. The behavior that occurred during LH stimulation was consistent with the only remaining possibility: the exploratory or appetitive phase of a complex behavior pattern which normally concludes with a consummatory response (Tinbergen, 1951). However, in the artificial conditions of the test cage, the behavior could not be consummated and the appetitive phase continued throughout the test period.

The exploration and self-stimulation data for the VMH group strongly support previous suggestions (Krasne, 1962; Lewinska \& Romaniuk, 1966) that VMH stimulation, at the parameters necessary to interrupt feeding, is aversive. Recent interpretations suggest that the VMH is not simply a "satiety" area which suppresses eating by inhibiting the LH, but that this area actually governs the long-term regulation of feeding (Davies, Nakajima, \& White, 1974; Panksepp, 1971). On the other hand, the original suppression-of-feeding reports (Anand \& Brobeck, 1951; Smith, 1956), as well as the effects of VMH stimulation in the present study, may have been the result of activating a general aversion system in the brain. The earlier reports may, in fact, have constituted the first demonstrations of the so-called medial aversion system proposed by Stein (1964).

\section{REFERENCES}

ANAND, B. K., \& Brobeck, J. R. Hypothalamic control of food intake in rats and cat. Yale Journal of Biology and Medicine, 1951, 24, 123-140.

BaLl, G. G. Self-stimulation in the ventromedial hypothalamus. Science, 1972, 178, 72-73.

BARnetT, S. A. The rat: $A$ study in behavior. Chicago: Aldine, 1963.

Berly ne, D. E. Curiosity and exploration. Science, 1966, 153, 25-33.

Blanchard, R. J., \& Blanchard, C. D. Defensive reactions in the albino rat. Learning and Motivation, 1971, 2, 351-362.

BLANCHARD, C. D., \& BLANCHARD, R. J. Innate and conditioned reactions to threat in rats with amygdaloid lesions. Journal of Comparative and Physiological Psychology, 1972, 81, 281-290.

Christopher, M., \& ButTer, C. M. Consummatory behaviors and locomotor exploration evoked from self-stimulation sites in rats. Journal of Comparative and Physiological Psychology, $1968,66,335-339$.

Davies, R., Nakajima, S., \& White, N. Enhancement of feeding produced by stimulation of the ventromedial hypothalamus. Journal of Comparative and Physiological Psychology, 1974, 86, 414-419.

DE GROOT, J. The rat forebrain in stereotaxic coordinates. Amsterdam: N.V. Noord-Hollandische, 1959. 
Glickman, S. E., \& Schiff, B. B. A biological theory of reinforcement. Psychological Review, 1967, 74, 81-109.

Goddard, V. Functions of the amygdala. Psychological Bulletin, 1964, 62, 89-109.

Goddard, V., McIntyre, D. C., \& Leech, K. A permanent change in brain function resulting from daily electrical stimulation. Experimental Neurology, 1969, 25, 295-330.

Grossman, S. P., \& Grossman, L. Food and water intake following lesions or electrical stimulation of the amygdala. American Journal of Physiology, 1963, 205, 761-765.

Halliday, M. S. Exploration and fear in the rat. In P. A. Jemell \& C. Loizos (Eds.), Play, exploration and territoriality in mammals. New York: Academic Press, 1966.

Hoebel, B. G., \& Teitelbaum, P. Hypothalamic control of feeding and self-stimulation. Science, 1962, 135, 375-377.

KAADA, B. R. Stimulation and regional ablation of the amygdaloid complex with reference to functional representation. In B. F. Eleftheriou (Ed.), The neurobiology of the amygdala. New York: Plenum, 1972.

Kemble, E. D., \& TAPP, J. J. Passive and active avoidance performance following small amygdaloid lesions in rats. Physiology and Behavior, 1968, 3, 713-718.

KRASNE, F. B. General disruption resulting from electrical stimulus of ventromedial hypothalamus. Science, 1962, 138, 822-823.

Lewinska, M. K., \& Romaniuk, A. Is the ventromedial nucleus of the hypothalamus a satiation center? Acta Biologiae Experimentalis (Warsaw), 1966, 26, 285-297.

Margules, D. L., \& Olds, J. Identical "feeding" and rewarding systems in the lateral hypothalamus of rats. Science, 1962, 135, 374-375.

McReynolds, P. Exploratory behavior: A theoretical interpretation. Psychological Reports, 1962, 11, 311-318.

Montgomery, K. C. The relation between fear induced by novel stimulation and exploratory behavior. Journal of Comparative Physiological Psychology, 1955, 48, 254-260.

PANKSEPP, J. Is satiety mediated by the ventromedial hypothalamus? Physiology and Behavior, 1971, 7, 381-384.
Pellegrino, L. Amygdaloid lesions and behavioral inhibition in the rat. Journal of Comparative and Physiological Psychology, 1968, 65, 483-491.

RACINE, R. J. Modification of seizure activity by electrical stimulation: I. After-discharge threshold. Electroencephalography and Clinical Neurophysiology, 1972, 32, 269-279.

REYNolds, R. W. The relationship between stimulation voltage and rate of hypothalamic self-stimulation in the rat. Journal of Comparative and Physiological Psychology, 1958, 51, 193-198.

SMITH, O. A. Stimulation of lateral and medial hypothalamus and food intake in the rat. Anatomical Record, 1956, 124, 363-364.

STEIN, L. Reciprocal action of reward and punishment mechanisms. In R. G. Heath (Ed.), The role of pleasure in behavior. New York: Harper, 1964.

TINBERGEN, N. The study of instinct. Oxford: Clarendon, 1951.

Valenstein, E. S., Cox, V. C., \& Kakolewski, J. W. Modification of motivated behavior elicited by electrical stimulation of the hypothalamus. Science, 1968, 159, 1119-1120.

WELKER, W. I. Escape, exploratory and food-seeking responses of rats in a novel situation. Journal of Comparative and Physiological Psychology, 1959, 52, 106-111.

WHITE, N. Self-stimulation and suppression of feeding observed at the same site in the amygdala. Physiology and Behavior, 1973, 10, 215-219.

White, N., \& Fisher, A. E. Relationship between amygdala and hypothalamus in the control of eating behavior. Physiology and Behavior, 1969, 4, 199-205.

WuRTz, R. H., \& Olds, J. Amygdaloid stimulation and operant reinforcement in the rat. Journal of Comparative and Physiological Psychology, 1963, 56, 941-949.

(Received for publication October 20, 1977; revision accepted December 21, 1977.) 